\title{
ON THE GENERATING FUNCTION OF A DOUBLY INFINITE, TOTALLY POSITIVE SEQUENCE
}

\author{
BY
}

ALBERT EDREI

Introduction. Let

$$
\cdots, a_{-2}, a_{-1}, a_{0}, a_{1}, a_{2}, \cdots,
$$

be a doubly infinite sequence with which we associate the generating Laurent series

$$
\sum_{\mu=-\infty}^{+\infty} a_{\mu} z^{\mu}=f(z) .
$$

We now consider the following definition due to Schoenberg [8; p. 362].

Definition 1. The sequence (1) is said to be totally positive if the fourway infinite matrix

$$
\begin{aligned}
& \cdots a_{2} \quad a_{1} \quad a_{0} \quad a_{-1} \quad a_{-2} \quad a_{-3} \quad a_{-4} \cdots \\
& \cdots a_{3} \quad a_{2} \quad a_{1} \quad a_{0} \quad a_{-1} \quad a_{-2} \quad a_{-3} \cdots \\
& \cdots a_{4} \quad a_{3} \quad a_{2} \quad a_{1} \quad a_{0} \quad a_{-1} \quad a_{-2} \cdots
\end{aligned}
$$

has only non-negative minors (of all finite orders, with any choice of rows and columns). Some of our statements will be simplified if we also introduce the following definition.

Definition 2. Consider the determinants

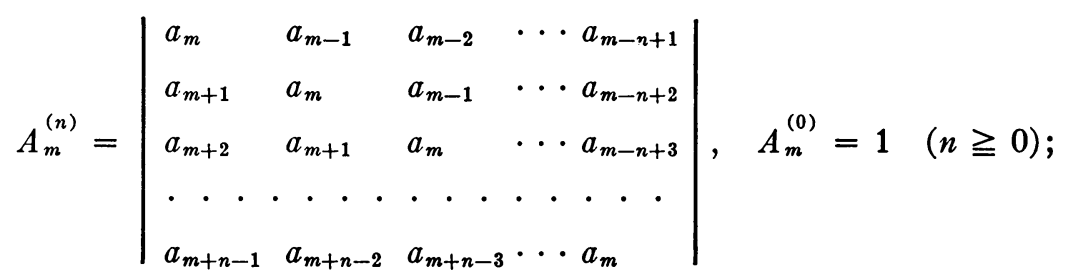

where $m$ is an integer, not necessarily positive. We say that (1) has the property (P) if $A_{m}^{(n)}>0$, for all $m$ and all $n \geqq 0$. Whenever we say that a Laurent series has the property $(\mathrm{P})$, we mean that the sequence of its coefficients has this property.

Received by the editors May 21, 1952. 
By a lemma of Fekete [7, p. 558], every sequence with the property $(\mathrm{P})$ is also totally positive in the following strict sense: all the finite minors of (3) are actually positive.

In the special case

$$
0=a_{-1}=a_{-2}=a_{-3}=\cdots
$$

the analytic character of the generating function $f(z)$ has been completely investigated [ 1 and 2 ], but the question of extending the results to the general case was left open.

We are now able to answer this question by proving that, in the general case, $f(z)$ has exactly the form conjectured by Schoenberg [8; p. 367]:

TheOREM. Let (1) be a totally positive sequence. If this sequence does not coincide with a sequence of the form

$$
\left\{a \rho^{\mu}\right\}_{\mu=-\infty}^{+\infty} \quad(a>0, \rho>0),
$$

then the Laurent series (2) converges in some ring

$$
r_{1}<|z|<r_{2} \quad\left(0 \leqq r_{1}<r_{2}\right),
$$

and the analytic continuation of (2) is of the form

$$
f(z)=C z^{\alpha} e^{c_{1} z+c_{-1} z^{-1}} \frac{\prod_{\nu=1}^{\infty}\left(1+\alpha_{\nu} z\right) \prod_{v=1}^{\infty}\left(1+\beta_{\nu} z^{-1}\right)}{\prod_{v=1}^{\infty}\left(1-\gamma_{\nu} z\right) \prod_{\nu=1}^{\infty}\left(1-\delta_{\nu} z^{-1}\right)},
$$

where $\kappa$ is an integer (not necessarily positive), $C \geqq 0, c_{1} \geqq 0, c_{-1} \geqq 0, \alpha_{\nu} \geqq 0$, $\beta_{v} \geqq 0, \gamma_{\nu} \geqq 0, \delta_{\nu} \geqq 0, \sum_{\nu=1}^{\infty}\left(\alpha_{\nu}+\beta_{\nu}+\gamma_{\nu}+\delta_{v}\right)<+\infty$.

1. Terminology and notation. From this point on, we adopt the following conventions. We say that (1) is normalized if

(i) its generating Laurent series (2) converges in the ring

$$
1 / R_{1}<|z|<R_{2}
$$

where $R_{1}>1, R_{2}>1$;

(ii) $f(z) \neq 0$, when $|z|=1$.

In our proofs, the special case

$$
a_{\nu}=a_{-\nu} \quad(\nu=1,2,3, \cdots),
$$

plays an important part; we shall refer to it as the symmetrical case. The abbreviation "n.t.p. sequence" stands for normalized, totally positive sequence.

By $C_{m}^{(n)}, E_{m}^{(n)}, \ldots$ we mean determinants obtained by replacing, in $A_{m}^{(n)}$, the $a$ 's, respectively, by $c$ 's, $e$ 's, . . .

We shall have to consider a number of convergent series, namely 


$$
\sum \epsilon_{\nu}, \quad \sum \zeta_{\nu}, \quad \sum \eta_{\nu}, \quad \sum \theta_{\nu},
$$

the summations are always to be extended from $\nu=1$ to $\nu=+\infty$. The terms of all these series are always non-negative and less than one.

By quasi-entire function we mean a single-valued function which is regular everywhere except perhaps at infinity anid at the origin. A quasi-meromorphic function is the ratio of two quasi-entire functions.

\section{Remarks on the property $(\mathrm{P})$.}

Lemma 1. Let (1) be normalized. To every $n(\geqq 1)$ there corresponds at least one integer $m$ (not necessarily positive) such that $A_{m}^{(n)} \neq 0$.

Proof. The series (2) does not vanish identically because $f(z) \neq 0$ when $|z|=1$. If the lemma were not true, there would exist a least integer $n(\geqq 1)$ such that

$$
A_{m}^{(n+1)}=0 \quad(m=0, \pm 1, \pm 2, \cdots) .
$$

The well known formula

$$
A_{m}^{(n+1)} A_{m}^{(n-1)}=\left\{A_{m}^{(n)}\right\}^{2}-A_{m+1}^{(n)} A_{m-1}^{(n)}
$$

would then yield

$$
\left\{A_{m}^{(n)}\right\}^{2}=A_{m+1}^{(n)} A_{m-1}^{(n)}
$$

which implies

$$
A_{m}^{(n)} \neq 0 \quad(m=0, \pm 1, \pm 2, \cdots),
$$

and also

$$
\frac{A_{m}^{(n)}}{A_{m-1}^{(n)}}=\frac{A_{m+1}^{(n)}}{A_{m}^{(n)}}=\omega \neq 0 .
$$

Hence

$$
\lim _{m \rightarrow \infty}\left|A_{m}^{(n)}\right|^{1 / m}=|\omega|,
$$

and a well known result of Hadamard implies

$$
|\omega| \leqq 1 / R_{2}^{n},
$$

where $R_{2}$ is the radius of convergence of

$$
\sum_{\nu=0}^{+\infty} a_{\nu} z^{\nu}
$$


Similarly

$$
\lim _{m \rightarrow+\infty}\left|A_{-m}^{(n)}\right|^{1 / m}=\frac{1}{|\omega|} \leqq \frac{1}{R_{1}^{n}},
$$

where $R_{1}$ is the radius of convergence of

$$
\sum_{\nu=0}^{+\infty} a_{-\nu} z^{\nu}
$$

Now (2.1) and (2.2) imply

$$
R_{1} R_{2} \leqq 1 .
$$

This contradicts (1.1) and our lemma is proved.

Let $f(z)$ and $g(z)$ be the generating Laurent series of the two normalized sequences $\left\{a_{\mu}\right\}_{\mu=-\infty}^{+\infty},\left\{b_{\mu}\right\}_{\mu=-\infty}^{+\infty}$. The sequence $\left\{c_{\mu}\right\}_{\mu=-\infty}^{+\infty}$ defined by

$$
c_{\mu}=\sum_{\sigma=-\infty}^{+\infty} a_{\mu-\sigma} b_{\sigma}
$$

is normalized and we obviously have

$$
f(z) g(z)=\sum_{\mu=-\infty}^{+\infty} c_{\mu} z^{\mu}
$$

Elementary computations easily yield.

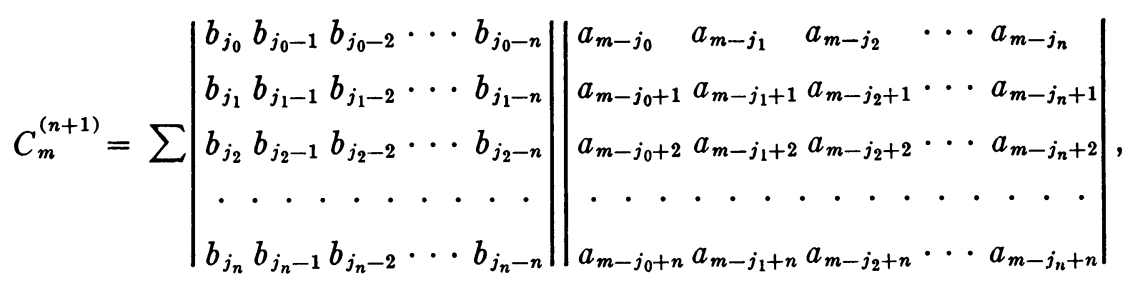

where the summation is to be extended over all distinct sets $\left(j_{0}, j_{1}, j_{2}, \cdots, j_{n}\right)$ of $n+1$ strictly increasing integers (not necessarily positive).

It is known that, if we put

$$
e^{\epsilon z}=\sum_{\nu=0}^{\infty} e_{\nu} z^{\nu}
$$

the determinants $E_{m}^{(n)}(m \geqq 0, n \geqq 1)$ are all positive $\left({ }^{1}\right)$. In view of $(2.3)$, this obviously implies that the Laurent expansion of $\exp \left[\epsilon\left(z+z^{-1}\right)\right]$ has the prop-

(1) The determinants $E$ are easy to compute explicitly. However, this computation may be avoided by observing, with Schoenberg, that the $E$ 's are not negative $[8$, p. 366$]$. As the Padé table of the exponential function is normal [6, p. 429], it is also clear that no $E$ can vanish. 
erty (P). Using this fact, (2.3) and Lemma 1, we easily obtain:

Lemma 2. Let $f(z)$ denote the Laurent expansion of a n.t.p. sequence, then the Laurent expansion of

$$
e^{\epsilon\left(z+z^{-1}\right)} f(z) \quad(\epsilon>0),
$$

has the property $(\mathrm{P})$.

3. The reciprocal of a Laurent series. Let (1) be normalized and consider its generating Laurent series (2). There will obviously exist $R_{3}(>1)$ such that both (2) and

$$
\frac{1}{f(z)}=\sum_{\mu=-\infty}^{+\infty} b_{\mu} z^{\mu}
$$

converge in the ring

$$
1 / R_{3}<|z|<R_{3} \text {. }
$$

Moreover, the $a$ 's and $b$ 's will be connected by the infinite system of equations

$$
\sum_{\mu=-\infty}^{+\infty} a_{\mu-\sigma} b_{\sigma}=\delta_{\mu 0} \quad(\mu=0, \pm 1, \pm 2, \cdots),
$$

where $\delta_{00}=1$ and $\delta_{\mu 0}=0$ if $\mu \neq 0$.

As suggested by the elementary theory of finite systems, we consider the determinant $A_{0}^{(2 n+1)}$ as well as the determinant $A_{0}^{(2 n+1)}(j, k)$ which is the minor obtained by deleting the $(n+1+j)$ th row and the $(n+1+k)$ th column of $A_{0}^{(2 n+1)}[-n \leqq j \leqq n ;-n \leqq k \leqq n]$.

With this notation, we obtain:

LeMma 3. Let (1) be a real, symmetrical normalized sequence; let (2) be its generating Laurent series, and assume $f(1)>0$. Then

$$
A_{0}^{(2 n+1)}>0 \quad(n=0,1,2,3, \cdots),
$$

and the b's are given by

$$
b_{k-j}=\lim _{n \rightarrow+\infty}(-1)^{k-j} \frac{A_{0}^{(2 n+1)}(j, k)}{A_{0}^{(2 n+1)}} .
$$

We first note that the assumptions of this lemma obviously imply that

$$
h(\phi)=f\left(e^{i \phi}\right)
$$

is positive for real values of $\phi$. Then, applying a theorem of the author [3], we obtain the formulae (3.2). 
Lemma 3 easily yields:

Lemma 4. Let the sequence (1) be normalized, totally positive, and symmetrical. Then the sequence $\left\{(-1)^{\mu} b_{\mu}\right\}_{\mu=-\infty}^{+\infty}$ defined by

$$
\frac{1}{f(-z)}=\sum_{u=-\infty}^{+\infty}(-1)^{\mu} b_{\mu} z^{\mu}
$$

is also normalized, totally positive, and symmetrical.

Proof. Let

$$
\left(r_{j k}^{(n)}\right)_{j, k=-n,-n+1,-n+2, \cdots, 0,1,2, \cdots, n}
$$

be the inverse of the matrix

$$
\left(a_{j-k}\right)_{j, k=-n,-n+1,-n+2, \cdots, 0,1,2, \cdots, n} .
$$

As observed by Aissen, Schoenberg, and Whitney [2], the total positivity of the matrix

$$
\left((-1)^{j+k} r_{j k}^{(n)}\right)_{j, k=-n,-n+1,-n+2, \cdots, 0,1,2, \cdots, n}
$$

follows from the total positivity of (3.3).

Now in view of (3.2), we have

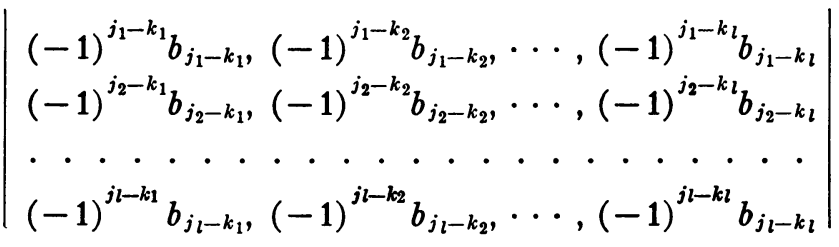

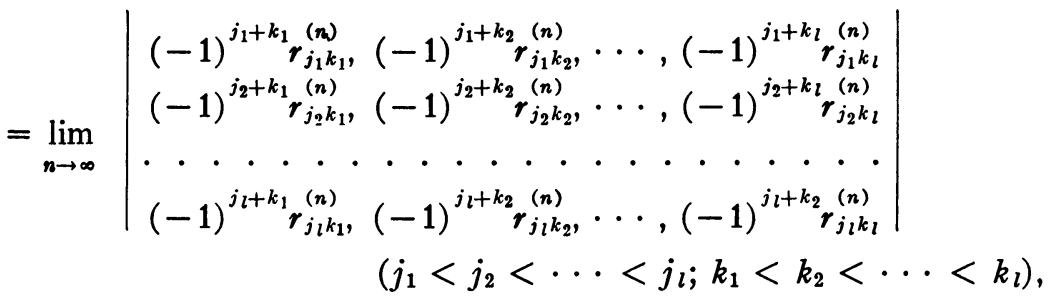

so that, if the matrix (3.3) is totally positive, the left-hand side of (3.4) is certainly not negative. Hence, the sequence $\left\{(-1)^{\mu} b_{\mu}\right\}_{\mu=-\infty}^{+\infty}$ is t.p.; as it is obviously normalized and symmetrical, our lemma is proved.

4. Poles of the generating function of a totally positive sequence. In this section we prove:

Lemma 5. Let (1) be a normalized sequence with the property (P). Consider the polynomials $Q^{(m, n)}(z)$ defined by 


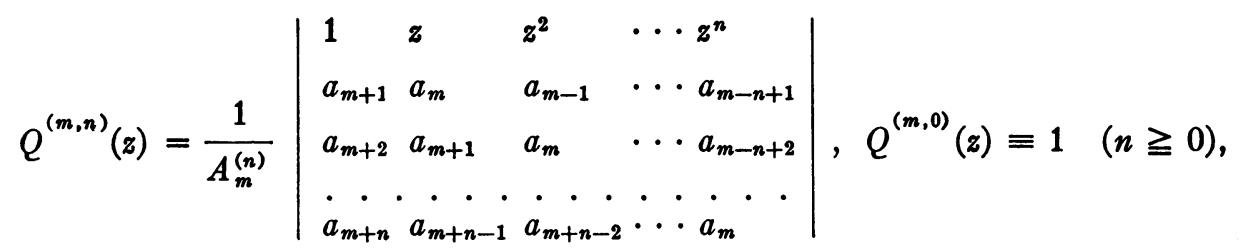

and also

$$
T^{(m, n)}(z)=Q^{(m, n)}(-z),
$$

where $m$ is an integer, not necessarily positive.

Then

(i) the coefficients of $T^{(m, n)}(z)$ are all positive;

$$
\lim _{m \rightarrow+\infty} Q^{(m, n)}(z)=Q^{(\infty, n)}(z)
$$

exists for all $n(\geqq 0)$;

(iii)

$$
\lim _{m \rightarrow+\infty} \frac{A_{m+1}^{(n+1)} / A_{m}^{(n+1)}}{A_{m+1}^{(n)} / A_{m}^{(n)}}=\zeta_{n+1}
$$

exists for all $n(\geqq 0)$;

(iv) the polynomials $Q^{(\infty, n)}(z)$ are connected by the recurrence relations

$$
Q^{(\infty, n+1)}(z)=\left(1-\zeta_{n+1} z\right) Q^{(\infty, n)}(z) \quad(n \geqq 0) ;
$$

(v) the Laurent series

$$
f(z) Q^{(\infty, n)}(z)=\sum_{\mu=-\infty}^{+\infty} p_{\mu}^{(n)} z^{\mu}
$$

has positive coefficients; it converges for

$$
\frac{1}{R_{1}}<|z|<\frac{1}{\zeta_{n+1}}
$$

(vi) $\sum \zeta_{\text {, converges and }}$

$$
\frac{1}{R_{2}}=\zeta_{1} \geqq \zeta_{2} \geqq \zeta_{3} \geqq \cdots ;
$$

(vii) The Laurent series

$$
\sum_{\mu=-\infty}^{+\infty} p_{\mu} z^{\mu}=f(z) \prod_{\nu=1}^{\infty}\left(1-\zeta_{\nu} z\right)
$$

converges for $|z|>1 / R_{1}$; the coefficients $p_{\mu}$ are non-negative. 
Proof. The proof of the lemma depends essentially on the identities $\left({ }^{2}\right)$

$$
\begin{aligned}
T^{(m, n+1)}(z) & =T^{(m+1, n)}(z)+z T^{(m, n)}(z) \frac{A_{m+1}^{(n+1)} / A_{m}^{(n+1)}}{A_{m+1}^{(n)} / A_{m}^{(n)}} \\
T^{(m+1, n+1)}(z)-T^{(m, n+1)}(z) & =-z T^{(m, n)}(z) \frac{A_{m+1}^{(n+2)} / A_{m}^{(n+1)}}{A_{m+1}^{(n+1)} / A_{m}^{(n)}} \\
& =-z \xi_{m n} T^{(m, n)}(z) .
\end{aligned}
$$

Assertion (i) is a consequence of Fekete's lemma [7, p. 558]. We now prove (ii). From (4.6), we obtain

$$
T^{(l+1, n+1)}(z)=T^{(m, n+1)}(z)-z \sum_{\nu=m}^{l} \xi_{\nu n} T^{(\nu, n)}(z) \quad\left(\xi_{\nu n}>0\right) .
$$

As the coefficients of the $T$ 's are positive, it follows from (4.7) that all the coefficients [except the constant term which is 1] decrease if we increase $l$ and keep $n$ fixed. This proves (ii) and also the convergence of $\sum_{\nu=1}^{\infty} \xi_{\nu n}$. Observing that, for a positive $z$,

$$
\lim _{m \rightarrow+\infty} z T^{(m, n)}(z)>0,
$$

we deduce from (4.5) the existence of the limit in (4.1). This proves (iii) and (iv) simultaneously.

To prove (v), we note that the coefficients of the Laurent expansion

$$
f(z) Q^{(m, n)}(z)=\sum_{\mu=-\infty}^{+\infty} p_{\mu}^{(m, n)} z^{\mu}
$$

are explicitly given by the formulae

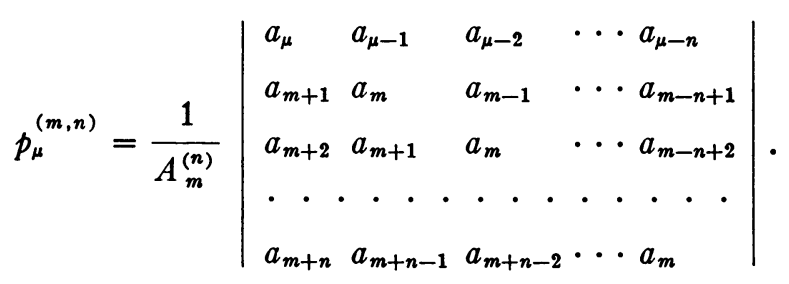

As (1) has the property (P), we have, by Fekete's lemma,

$$
p_{\mu}^{(m, n)}>0
$$

$$
(\mu \leqq m) \text {. }
$$

(2) These formulae are straightforward consequences of Jacobi's theorem on the minors of the adjoint of a given determinant. 
For $n$ fixed and $m \rightarrow+\infty$,

$$
f(z) Q^{(m, n)}(z) \rightarrow f(z) Q^{(\infty, n)}(z)=\sum_{\mu=-\infty}^{+\infty} p_{\mu}^{(n)} z^{\mu},
$$

uniformly in

$$
\frac{1}{R_{1}^{*}} \leqq|z| \leqq R_{2}^{*} \quad\left(1<R_{1}^{*}<R_{1} ; 1<R_{2}^{*}<R_{2}\right) .
$$

Now (4.7) implies

$$
f(z) Q^{(\infty, n)}(z)=f(z) Q^{(m, n)}(z)+z \sum_{\nu=m}^{+\infty} \xi_{\nu, n-1}\left\{f(z) Q^{(v, n-1)}(z)\right\},
$$

from which we deduce

$$
p_{\mu}^{(n)}=p_{\mu}^{(m, n)}+\sum_{\nu=m}^{+\infty} \xi_{\nu, n-1} p_{\mu-1}^{(\nu, n-1)} \quad(\mu=0, \pm 1, \pm 2, \cdots) .
$$

In view of (4.9)

$$
p_{\mu}^{(n)}>p_{\mu}^{(m, n)}>0
$$$$
(\mu \leqq m),
$$

so that the coefficients of (4.3) are all positive. To find the region of convergence of $(4.3)$, we observe that

$$
p_{m+1}^{(m, n)}=0
$$

so that, using (4.10) and (4.11), we also obtain

$$
p_{m+1}^{(n)}<p_{m}^{(n-1)}\left(\xi_{m, n-1}+\xi_{m+1, n-1}+\xi_{m+2, n-1}+\cdots\right) \quad(n \geqq 1) .
$$

Putting

$$
\limsup _{m \rightarrow+\infty}\left|\left(p_{m}^{(n)}\right)^{1 / m}\right|=\sigma_{n} \quad(n=0,1,2,3, \cdots)
$$

we deduce from (4.12)

$$
\sigma_{n} \leqq \sigma_{n-1} \limsup _{m \rightarrow+\infty}\left|\left(\sum_{\nu=m}^{+\infty} \xi_{\nu, n-1}\right)^{1 / m}\right| .
$$

Moreover, as all series of the form $\sum_{\nu=1}^{+\infty} \xi_{\nu, n-1}$ are convergent, (4.14) trivially implies

$$
\sigma_{n} \leqq \sigma_{n-1}
$$

We now verify the inequalities 


$$
\sigma_{k} \leqq \zeta_{k+1}
$$$$
(k=0,1,2, \cdots) \text {, }
$$

by an induction over $k$.

We note that $p_{\mu}^{(0)}=a_{\mu}$ implies $\sigma_{0}=\zeta_{1}$, and assume that (4.16) is true for $k=0,1,2, \cdots,(n-1)$. We distinguish two cases,

$$
\begin{aligned}
& \zeta_{1} \zeta_{2} \cdots \zeta_{n}=0 \\
& \zeta_{1} \zeta_{2} \cdots \zeta_{n} \neq 0
\end{aligned}
$$

The case (a) is immediately settled by observing that our induction assumption and (4.15) then yield $\sigma_{k}=0$, for $k \geqq n$.

In the case (b), we observe that

$$
\lim _{m \rightarrow+\infty}\left(\sum_{\nu=m}^{+\infty} \xi_{\nu, n-1}\right)^{1 / m} \leqq \frac{\zeta_{n+1}}{\zeta_{n}}
$$

is trivial if $\zeta_{n+1} \geqq \zeta_{n}$.

If $\zeta_{n+1}<\zeta_{n}$, we return to the formulae (4.6) which define the quantities $\xi_{m n}$ as ratios of determinants.

Observing that (4.1) implies

$$
\lim _{m \rightarrow+\infty} \frac{A_{m+1}^{(n+1)}}{A_{m}^{(n+1)}}=\zeta_{1} \zeta_{2} \cdots \zeta_{n} \zeta_{n+1}, \quad \lim _{m \rightarrow+\infty}\left|\left(A_{m}^{(n)}\right)^{1 / m}\right|=\zeta_{1} \zeta_{2} \cdots \zeta_{n},
$$

we obtain

$$
\lim _{m \rightarrow+\infty}\left|\left(\xi_{m, n-1}\right)^{1 / m}\right|=\lim _{m \rightarrow+\infty}\left|\left(\frac{A_{m+1}^{(n+1)} A_{m}^{(n-1)}}{A_{m+1}^{(n)} A_{m}^{(n)}}\right)^{1 / m}\right|=\frac{\zeta_{n+1}}{\zeta_{n}} .
$$

We may then compare $\sum_{v=m}^{\infty} \xi_{v, n-1}$ to the remainder of a geometric series. This comparison leads again to (4.17). Combining (4.14) and (4.17) we complete the induction which proves (4.16) and, therefore, (v).

To prove (vi), we observe that (4.2) implies

$$
p_{\mu}^{(n+1)}=p_{\mu}^{(n)}-\zeta_{n+1} p_{\mu-1}^{(n)}>0,
$$

so that the Laurent expansion of $f(z) Q^{(\infty, n+1)}(z)$ is majorized by the Laurent expansion of $f(z) Q^{(\infty, n)}(z)$. This obviously yields

$$
\left|f(-1) Q^{(\infty, n)}(-1)\right| \leqq f(1),
$$

or also

$$
\left(1+\zeta_{1}\right)\left(1+\zeta_{2}\right) \cdots\left(1+\zeta_{n}\right) \leqq \frac{f(1)}{|f(-1)|} .
$$

Now $f(-1) \neq 0$ because (1) is normalized, and (4.19) is true for any positive 
integer $n$. This proves the convergence of $\sum \zeta_{\nu}$.

In order to verify $(4.4)$, we note that $(4.18)$ also implies

$$
\zeta_{n+1} \leqq \sigma_{n},
$$

which, combined with (4.16), yields

$$
\zeta_{n+1}=\sigma_{n} .
$$

Hence (4.4) coincides with (4.15). Assertion (vii) follows immediately from (v) and (vi).

LEMMA 6. If (2) is the generating Laurent series of a normalized totally positive sequence, there exist two entire functions $Q_{1}(z), Q_{2}(z)$ of the forms

$$
\begin{array}{ll}
Q_{1}(z)=\prod_{\nu=1}^{\infty}\left(1-\zeta_{\nu} z\right) & \left(0 \leqq \zeta_{\nu} \leqq \frac{1}{R_{2}}<1 ; \sum \zeta_{\nu}<+\infty\right), \\
Q_{2}(z)=\prod_{\nu=1}^{\infty}\left(1-\epsilon_{\nu} z\right) & \left(0 \leqq \epsilon_{\nu} \leqq \frac{1}{R_{1}}<1 ; \sum \epsilon_{\nu}<+\infty\right),
\end{array}
$$

and such that

$$
g(z)=f(z) Q_{1}(z) Q_{2}(1 / z)
$$

is quasi-entire. Moreover, $g(z)>0$ for all positive values of $z$.

Proof. By Lemma 2, the expansions of

$$
e^{z+z^{-1}} f(z) \text { and } e^{z+z^{-1}} f(1 / z)
$$

both have the property $(\mathrm{P})$. Hence Lemma 5 proves the existence of two entire functions $Q_{1}(z)$ and $Q_{2}(z)$ such that

(i) $e^{z+z^{-1}} f(z) Q_{1}(z)$ is regular for $|z|>1 / R_{1}$,

(ii) $e^{z+z^{-1}} f(1 / z) Q_{2}(z)$ is regular for $|z|>1 / R_{2}$.

Now (ii) implies that $Q_{2}(1 / z) f(z)$ is regular for $0<|z|<R_{2}$ so that $g(z)$ is regular for $0<|z|<R_{2}$. Similarly (i) shows that $g(z)$ is regular for $|z|>1 / R_{1}$. Hence $g(z)$ is quasi-entire. Now

$$
\begin{aligned}
Q_{1}(z)>0 & \text { if } z \leqq 1, \\
Q_{2}(1 / z)>0 & \text { if } z \geqq 1 .
\end{aligned}
$$

As the coefficients of the Laurent expansions of both $e^{z+z^{-1}} f(z) Q_{1}(z)$ and $e^{z+z^{-1}} f(z) Q_{2}(1 / z)$ are non-negative, we obtain

$$
\begin{array}{lr}
{\left[e^{z+z^{-1}} f(z) Q_{1}(z)\right] Q_{2}(1 / z)>0} & \text { for } z \geqq 1, \\
{\left[e^{z+z^{-1}} f(z) Q_{2}(1 / z)\right] Q_{1}(z)>0} & \text { for } 0<z \leqq 1 .
\end{array}
$$

Hence $g(z)>0$ if $z>0$.

5. Zeros of the generating function of a totally positive sequence. 
Lemma 7. Let $\phi(z)$ denote the generating Laurent series of a symmetrical, normalized, totally positive sequence. Then the analytic continuation of $\phi(z)$ is of the form

$$
z^{\alpha} e^{\psi(z)} \prod_{\nu=1}^{\infty} \frac{\left(1+\eta_{\nu} z\right)\left(1+\eta_{\nu} z^{-1}\right)}{\left(1-\theta_{\nu} z\right)\left(1-\theta_{\nu} z^{-1}\right)},
$$

where $\psi(z)$ is a quasi-enture function, $\kappa$ is an integer (not necessarily positive) $0 \leqq \eta_{\nu}<1,0 \leqq \theta_{v}<1, \sum\left(\eta_{v}+\theta_{v}\right)<+\infty$.

Proof. By Lemma 4 the Laurent series $1 / \phi(-z)$ generates a symmetrical, normalized, totally positive sequence. Applying Lemma 6 to each of the two series, $\phi(z)$ and $1 / \phi(-z)$, we verify the existence of

$$
P(z)=\prod_{\nu=1}^{\infty}\left(1-\eta_{\nu} z\right), \quad Q(z)=\prod_{\nu=1}^{\infty}\left(1-\theta_{\nu} z\right),
$$

such that each of the two functions

$$
\phi(z) Q(z) Q(1 / z), \quad \frac{P(z) P(1 / z)}{\phi(-z)}
$$

is quasi-entire. Now Lemma 6 also shows that the latter function does not vanish for $z>0$, so that

$$
\frac{\phi(z)}{P(-z) P(-1 / z)}
$$

is regular in the neighborhood of the negative axis. Hence

$$
\Lambda(z)=\phi(z) \frac{Q(z) Q(1 / z)}{P(-z) P(-1 / z)}
$$

is quasi-entire. The same arguments show that $1 / \Lambda(-z)$ is quasi-entire, so that $\Lambda(z)$ does not vanish in the region

$$
0<|z|<+\infty \text {. }
$$

Hence $\Lambda(z)$ is of the form

$$
z^{\kappa} e^{\psi(z)},
$$

where $\psi(z)$ is quasi-entire; this proves Lemma 7.

Lemma 8. Let $f(z)$ denote the generating Laurent series of a n.t.p. sequence. Then the analytic continuation of $f(z)$ is necessarily of the form

$$
\begin{gathered}
z^{\alpha} e^{\psi(z)} \prod_{\nu=1}^{\infty} \frac{\left(1+\alpha_{\nu} z\right)\left(1+\beta_{\nu} z^{-1}\right)}{\left(1-\gamma_{\nu} z\right)\left(1-\delta_{\nu} z^{-1}\right)} \\
\left(0 \leqq \alpha_{\nu}<1,0 \leqq \beta_{\nu}<1,0 \leqq \gamma_{\nu}<1,0 \leqq \delta_{\nu}<1 ; \sum\left(\alpha_{\nu}+\beta_{\nu}+\gamma_{\nu}+\delta_{\nu}\right)<\infty\right),
\end{gathered}
$$


where $\psi(z)$ is quasi-entire and $\kappa$ is an integer. If, in the definition of a normalized t.p. sequence, we omit condition (ii) of $\$ 1$, the analytic continuation of $f(z)$ either vanishes identically or else is still of the form (5.1). However, in the latter case, the inequalities

$$
\alpha_{\nu}<1, \quad \beta_{\nu}<1, \quad \gamma_{\nu}<1, \quad \delta_{\nu}<1
$$

are not necessarily true.

Proof. We first assume that (1) is n.t.p. Then, by Lemma 6, we know that the analytic continuation of $f(z)$ is of the form

$$
\frac{g(z)}{\prod_{\nu=1}^{\infty}\left(1-\zeta_{\nu} z\right) \prod_{\nu=1}^{\infty}\left(1-\epsilon_{\nu} z^{-1}\right)}
$$

where $g(z)$ is quasi-entire. Now $f(z) f(1 / z)$ generates a symmetrical n.t.p. (cf. [8, pp. 365-366]), so that Lemma 7 implies

$$
\begin{aligned}
& \frac{g(z) g(1 / z)}{\prod_{\nu=1}^{\infty}\left(1-\zeta_{\nu} z\right)\left(1-\zeta_{\nu} z^{-1}\right)\left(1-\epsilon_{\nu} z^{-1}\right)\left(1-\epsilon_{\nu} z\right)} \\
& =z^{\kappa} e^{\psi(z)} \prod_{\nu=1}^{\infty} \frac{\left(1+\eta_{\nu} z\right)\left(1+\eta_{\nu} z^{-1}\right)}{\left(1-\theta_{\nu} z\right)\left(1-\theta_{\nu} z^{-1}\right)} .
\end{aligned}
$$

Hence the roots of $g(z)$ are to be found among the roots of the function

$$
\prod_{\nu=1}^{\infty}\left(1-\zeta_{\nu} z\right)\left(1-\zeta_{\nu} z^{-1}\right)\left(1-\epsilon_{\nu} z\right)\left(1-\epsilon_{\nu} z^{-1}\right)\left(1+\eta_{\nu} z\right)\left(1+\eta_{\nu} z^{-1}\right) .
$$

As $g(z)>0$ when $z>0$, we see that these roots are also negative; this proves the first part of Lemma 8 . The other statements of the lemma trivially follow from this first part.

\section{Determination of the exponential factor.}

Lемма 9. Let (1) be a n.t.p. sequence. Then the analytic continuation of $f(z)$ is of the form (5.1), where the Laurent expansion of the quasi-entire function $\psi(z)$ is of the form

$$
\psi(z)=q_{1} z+q_{-1} z^{-1}+\sum_{\mu=-\infty}^{+\infty} q_{2 \mu} z^{2 \mu} .
$$

Proof. As (1) is a n.t.p. sequence, the coefficients of the Laurent expansion

$$
\sum_{\mu=-\infty}^{+\infty} a_{2 \mu} z^{\mu}=f^{*}(z)
$$

form a totally positive sequence. Moreover, $\left\{a_{2 \mu}\right\}_{\mu=-\infty}^{+\infty}$ is certainly n.t.p. except if $f^{*}(z)$ vanishes on the unit circle. Hence, by Lemma 8 , we know that 
the analytic continuation of $f^{*}(z)$ is of the form

$$
C z^{\kappa^{*}} e^{\nu^{*}(z)} \frac{\prod_{\nu=1}^{\infty}\left(1+\alpha_{\nu}^{*} z\right)\left(1+\beta_{\nu}^{*} z^{-1}\right)}{\prod_{\nu=1}^{\infty}\left(1-\gamma_{\nu}^{*} z\right)\left(1-\delta_{\nu}^{*} z^{-1}\right)}
$$

where $\kappa^{*}$ is an integer, $\psi^{*}(z)$ a quasi-entire function, $C \geqq 0, \alpha_{\nu}^{*} \geqq 0, \beta_{\nu}^{*} \geqq 0$, $\gamma_{\nu}^{*} \geqq 0, \delta_{\nu}^{*} \geqq 0, \quad \sum\left(\alpha_{\nu}^{*}+\beta_{\nu}^{*}+\gamma_{\nu}^{*}+\delta_{\nu}^{*}\right)<+\infty$. On the other hand, applying directly Lemma 8 to $f(z)$, we see that the analytic continuation of $f(z)$ is of the form (5.1). Hence

$$
\frac{f(z)+f(-z)}{2}=f^{*}\left(z^{2}\right)
$$

implies

$$
e^{\psi(z)} \frac{\prod_{\nu=1}^{\infty}\left(1+\alpha_{\nu} z\right)\left(1+\beta_{\nu} z^{-1}\right)}{\prod_{\nu=1}^{\infty}\left(1-\gamma_{\nu} z\right)\left(1-\delta_{\nu} z^{-1}\right)}
$$

$$
\begin{aligned}
& +(-1)^{\kappa} e^{\psi(-z)} \frac{\prod_{\nu=1}^{\infty}\left(1-\alpha_{\nu} z\right)\left(1-\beta_{\nu} z^{-1}\right)}{\prod_{\nu=1}^{\infty}\left(1+\gamma_{\nu} z\right)\left(1+\delta_{\nu} z^{-1}\right)} \\
& =2 C e^{\psi *\left(z^{2}\right)} z^{2 \kappa^{*-1}} \frac{\prod_{\nu=1}^{\infty}\left(1+\alpha_{\nu}^{*} z^{2}\right)\left(1+\beta_{\nu}^{*} z^{-2}\right)}{\prod_{\nu=1}^{\infty}\left(1-\gamma_{\nu}{ }^{*} z^{2}\right)\left(1-\delta_{\nu}^{*} z^{-2}\right)} .
\end{aligned}
$$

Put

$$
\psi(z)=\sum_{\mu=-\infty}^{+\infty} q_{\mu} z^{\mu}
$$

(6.5) $u(z)=\exp \left(2 \sum_{\mu=-\infty}^{+\infty} q_{2 \mu+1} z^{2 \mu+1}\right) \frac{\prod_{\nu=1}^{\infty}\left(1+\alpha_{\nu} z\right)\left(1+\beta_{\nu} z^{-1}\right)\left(1+\gamma_{\nu} z\right)\left(1+\delta_{\nu} z^{-1}\right)}{\prod_{\nu=1}^{\infty}\left(1-\alpha_{\nu} z\right)\left(1-\beta_{\nu} z^{-1}\right)\left(1-\gamma_{\nu} z\right)\left(1-\delta_{\nu} z^{-1}\right)}$.

(6.4) is then equivalent to

$$
\begin{aligned}
u(z)=(-1)^{\kappa}=2 C z^{2 \kappa^{*}-\kappa} & \\
& \cdot \exp \left(\psi\left(z^{2}\right)-\psi(-z)\right) \prod_{\nu=1}^{\infty} \frac{\left(1+\alpha_{\nu}{ }^{*} z^{2}\right)\left(1+\beta_{\nu}^{*} z^{-2}\right)\left(1+\gamma_{\nu} z\right)\left(1+\delta_{\nu} z^{-1}\right)}{\left(1-\gamma_{\nu}{ }^{*} z^{2}\right)\left(1-\delta_{\nu}{ }^{*} z^{-2}\right)\left(1-\alpha_{\nu} z\right)\left(1-\beta_{\nu} z^{-1}\right)} .
\end{aligned}
$$

This relation, together with (6.5), gives us complete information on the distribution of the values $0, \infty,(-1)^{x}$ of the quasi-meromorphic function $u(z)$.

Consider the zeros and the poles of $u(z)$ which lie outside the unit circle; they are all real and their exponent of convergence does not exceed 1 . The function $u(z)+(-1)^{\kappa}$, if it does not vanish identically, has all its zeros on the real and on the imaginary axes. Hence by a result of the author $[4], u(z)$ 
has, at infinity, an essential singularity of order not exceeding two $\left({ }^{3}\right)$, and therefore

$$
0=q_{3}=q_{5}=q_{7}=\cdots .
$$

The same arguments may be applied to $f(1 / z)$, instead of $f(z)$, and lead to

$$
0=q_{-3}=q_{-5}=q_{-7}=\cdots .
$$

In the trivial case

$$
u(z)+(-1)^{\kappa} \equiv 0,
$$

$f(z)$ must be an odd function so that, in view of the total positivity of (1), its Laurent expansion reduces to a single term.

Lемма 10. Under the assumption of Lemma 9, and with the same notation, we also have

$$
q_{2 \nu}+q_{-2 \nu}=0 \quad(\nu=1,2,3, \cdots) .
$$

Proof. By Lemma 9,

$$
e^{z+z^{-1}} f(z) f\left(\frac{1}{z}\right)=\exp \left\{v_{1}\left(z+z^{-1}\right)+\sum_{\nu=0}^{\infty} v_{2 \nu}\left(z^{2 \nu}+z^{-2 \nu}\right)\right\} \Pi(z),
$$

where

$$
v_{2 \nu}=v_{-2 \nu}=q_{2 \nu}+q_{-2 \nu} \quad(\nu=0,1,2, \cdots)
$$

and

$$
\Pi(z)=\prod_{\nu=1}^{\infty} \frac{\left(1+\alpha_{\nu} z\right)\left(1+\alpha_{\nu} z^{-1}\right)\left(1+\beta_{\nu} z\right)\left(1+\beta_{\nu} z^{-1}\right)}{\left(1-\gamma_{\nu} z\right)\left(1-\gamma_{\nu} z^{-1}\right)\left(1-\delta_{\nu} z\right)\left(1-\delta_{\nu} z^{-1}\right)}
$$

The Laurent expansions of $f(z)$ and $f(1 / z)$ both generate n.t.p. sequences so that the same is true of the expansion of $f(z) f(1 / z)$. Moreover, this expansion is obviously symmetrical so that, by Lemma 4 , the expansion of

$$
1 / f(-z) f(-1 / z)
$$

also generates a n.t.p. sequence. Hence, by Lemma 2, the Laurent expansions of the two functions

$$
e^{\left(z+z^{-1}\right)} f(z) f(1 / z), \quad e^{\left(z+z^{-1}\right)} / f(-z) f(-1 / z)
$$

both have the property $(\mathrm{P})$. We may apply Lemma 5 to the sequences generated by these two expansions; hence, by assertion (vii) of this lemma, we

(3) The same conclusion may be reached by (i) restating a classical result of Nevanlinna $[5$, p. 72] for functions meromorphic in the neighborhood of $z=\infty$ (but not necessarily meromorphic in the whole plane) and (ii) observing that, outside the unit circle, the roots of $u(z)+(-1)^{\kappa}=0$ have an exponent of convergence not exceeding two. 
conclude that the suitable Laurent expansions of

$$
\begin{aligned}
u_{1}(z)=\exp \left\{v_{1}\left(z+z^{-1}\right)+\sum_{\nu=0}^{+\infty} v_{2 \nu}\left(z^{2 \nu}+z^{-2 \nu}\right)\right\} \\
\cdot \prod_{\nu=1}^{\infty} \frac{\left(1+\alpha_{\nu} z\right)\left(1+\alpha_{\nu} z^{-1}\right)\left(1+\beta_{\nu} z\right)\left(1+\beta_{\nu} z^{-1}\right)}{\left(1-\gamma_{\nu} z^{-1}\right)\left(1-\delta_{\nu} z^{-1}\right)}
\end{aligned}
$$

and

$$
\begin{aligned}
u_{2}(z)=\exp \left\{v_{1}\left(z+z^{-1}\right)-\sum_{\nu=0}^{+\infty} v_{2 \nu}\left(z^{2 \nu}+z^{-2 \nu}\right)\right\} & \\
& \cdot \prod_{\nu=1}^{\infty} \frac{\left(1+\gamma_{\nu} z\right)\left(1+\gamma_{\nu} z^{-1}\right)\left(1+\delta_{\nu} z\right)\left(1+\delta_{\nu} z^{-1}\right)}{\left(1-\alpha_{\nu} z^{-1}\right)\left(1-\beta_{\nu} z^{-1}\right)}
\end{aligned}
$$

converge for $|z| \geqq 1$ and have non-negative coefficients.

Hence, putting

$$
M_{1}(r)=\max _{|z|=r}\left|u_{1}(z)\right| \quad(r>1) ; \quad M_{2}(r)=\max _{|z|=r}\left|u_{2}(z)\right| \quad(r>1),
$$

we have $M_{1}(r)=u_{1}(r), M_{2}(r)=u_{2}(r)$, and

$$
\begin{aligned}
& \quad M_{1}(r) M_{2}(r)=\exp \left\{2 \nu_{1}\left(r+r^{-1}\right)\right\} \\
& \prod_{\nu=1}^{\infty} \frac{\left(1+\alpha_{\nu} r\right)\left(1+\alpha_{\nu} r^{-1}\right)\left(1+\beta_{\nu} r\right)\left(1+\beta_{\nu} r^{-1}\right)\left(1+\gamma_{\nu} r\right)\left(1+\gamma_{\nu} r^{-1}\right)\left(1+\delta_{\nu} r\right)\left(1+\delta_{\nu} r^{-1}\right)}{\left(1-\alpha_{\nu} r^{-1}\right)\left(1-\beta_{\nu} r^{-1}\right)\left(1-\gamma_{\nu} r^{-1}\right)\left(1-\delta_{\nu} r^{-1}\right)} .
\end{aligned}
$$

As the coefficients of the expansion of $u_{2}(r)$ are non-negative, there obviously exists an integer $\lambda$, and a positive constant $L$, such that

$$
M_{2}(r) r^{\lambda} \geqq L \quad(r>1) .
$$

Hence, by (6.11), the increase of $M_{1}(r)$ is dominated by the increase of an entire function of order 1 . This implies

$$
0=v_{2}=v_{4}=v_{6}=\cdots,
$$

and in view of (6.9), our lemma is proved.

Lemma 11. Under the assumptions of Lemma 9, and with the same notation, we also have

$$
\begin{gathered}
q_{2 \nu}=q_{-2 \nu}=0 \\
q_{1} \geqq 0, \quad q_{-1} \geqq 0 .
\end{gathered}
$$

Proof. The Laurent expansions of the two functions

$$
e^{\epsilon\left(z+z^{-1}\right)} f(z) \quad(\epsilon>0), \quad e^{\epsilon\left(z+z^{-1}\right)} f(1 / z) \quad(\epsilon>0)
$$

have the property $(\mathrm{P})$ [by Lemma 2]. We may now argue, with these two 
functions, exactly as we argued, in Lemma 10, with the two functions (6.10). In view of (6.7) we shall obtain a formula analogous to (6.11). The conclusion which corresponds to (6.12) is now (6.13).

It is easily verified that $q_{1}$ and $q_{-1}$ must be real and $e^{q_{0}}>0$. Hence it suffices to show the impossibility of

$$
q_{1}<0, \quad q_{-1}<0 .
$$

If the first of these inequalities were true, we could find $\epsilon(>0)$ such that

$$
q_{1}+\epsilon<0
$$

hence for $z>0$, and for every positive $\tau$, we would have

$$
\lim _{z \rightarrow+\infty} z^{\tau} F(z)=0,
$$

where

$$
\begin{aligned}
F(z) & =e^{\epsilon\left(z+z^{-1}\right)} f(z) \prod_{\nu=1}^{\infty}\left(1-\gamma_{\nu} z\right) \\
& =z^{\kappa} \exp \left(\left(q_{1}+\epsilon\right) z+\left(q_{-1}+\epsilon\right) z^{-1}\right) \frac{\prod_{\nu=1}^{\infty}\left(1+\alpha_{\nu} z\right)\left(1+\beta_{\nu} z^{-1}\right)}{\prod_{\nu=1}^{\infty}\left(1-\delta_{\nu} z^{-1}\right)} .
\end{aligned}
$$

By Lemma 5, the coefficients of the Laurent expansion of $F(z)$ are nonnegative, so that (6.17) would imply $F(z) \equiv 0$. This is impossible because (1) is normalized. Arguing with $f(1 / z)$ instead of $f(z)$, we also have $q_{-1} \geqq 0$, and Lemma 11 is completely proved.

Schoenberg has shown that the only t.p. sequences which cannot be normalized by a substitution $z \mid \chi z(\chi>0)$ are of the form (6). Hence our theorem trivially follows from Lemma 11.

\section{REFERENCES}

1. M. Aissen, A. Edrei, I. J. Schoenberg, and A. Whitney, On the generating functions of totally positive sequences, Proc. Nat. Acad. Sci. U. S. A. vol. 37 (1951) pp. 303-307.

2. M. Aissen, I. J. Schoenberg, and A. Whitney, On the generating functions of totally positive sequences (to appear in the Journal d'Analyse Mathématique).

3. A. Edrei and G. Szegö, A note on the reciprocal of a Fourier series (to appear in the Proceedings of the American Mathematical Society).

4. A. Edrei, Meromorphic functions with three radially distributed values (not yet published).

5. R. Nevanlinna, Le therème de Picard-Borel et la théorie des fonctions meromorphes, Paris, 1929.

6. O. Perron, Die Lehre von den Kettenbrïchen, 2d ed., Leipzig, 1929.

7. I. J. Schoenberg, Zur Abzählung der reellen Wurzeln algebraischer Gleichungen, Math. Zeit. vol. 38 (1934) pp. 546-564.

8. - Some analytical aspects of the problem of smoothing, Courant Anniversary Volume, New York, 1948, pp. 351-370.

UNIVERSiTY OF Colorado, BOUlder, Colo. 